\title{
Relação de fungos micorrízicos arbusculares e rizobactérias no crescimento de mudas de oliveira (Olea europaea)
}

\author{
Relationship of arbuscular mycorrhizal fungi and rhizobacteria on the growth of olive \\ tree seedlings (Olea europaea)
}

Samara Maria Lopes CostaI, Rogério Melloni ${ }^{\mathrm{II}}$

\begin{abstract}
Resumo
Inúmeros fatores influenciam a produtividade da oliveira (Olea europaea L.), desde climáticos até o manejo adequado da espécie. A eficiência da utilização de insumos microbiológicos, como fungos micorrízicos arbusculares e rizobactérias, tem sido registrada, principalmente na literatura internacional. No Brasil, os estudos ainda são incipientes. Diante disso, o objetivo deste trabalho foi analisar o efeito da coinoculação de fungos micorrízicos arbusculares e rizobactérias no crescimento de mudas de cultivares de oliveira. Conduziram-se dois experimentos simultaneamente em DIC, em esquema fatorial $(3 \times 3+1)$ por cultivar de oliveira (Arbequina e Maria da Fé), sendo 3 isolados de rizobactérias Pseudomonas sp, Paenibacillus sp1, Paenibacillus sp2, combinados com 3 espécies de FMAs (Acaulospora scrobiculata, Gigaspora rosea e Rhizophagus clarus) e um controle total (sem fungo e sem bactéria), com três repetições por tratamento, totalizando 60 unidades experimentais. Foram avaliados, após 12 meses, a altura, diâmetro, número de folhas, matéria fresca de raiz, matéria seca da parte aérea, teor e acúmulo de $\mathrm{N}$ e $\mathrm{P}$, intensidade e porcentagem colonização radicular, micélio extrarradicular ativo e total e número de esporos. Houve resultados diferenciados dos tratamentos para cada cultivar, constatando-se que, para alguns atributos houve efeito isolado do FMA ou da coinoculação de FMA e rizobactéria, que proporcionaram aumentos significativos no crescimento e desenvolvimento das mudas de cultivares de oliveira.
\end{abstract}

Palavras-chave: Bactérias promotoras de crescimento; Insumos microbiológicos; Micorriza

\begin{abstract}
A number of factors influence olive tree productivity (Olea europaea L.), from climatic factors to proper management of the species. The efficiency of using microbiological inputs, such as arbuscular mycorrhizal fungi and rhizobacteria, have been registered, mainly at the international literature. In Brazil, studies are still incipient. Therefore, the objective of this work was to analyze the effect of the co-inoculation of arbuscular mycorrhizal fungi and rhizobacteria on the growth of olive tree seedlings. Two experiments were conducted simultaneously in DIC, in a factorial scheme $(3 \times 3+1)$ by olive cultivar (Arbequina and Maria da Fé), three rhizobacterial isolates Pseudomonas sp, Paenibacillus sp1, and Paenibacillus sp2 were combined with three species of AMF (Acaulospora scrobiculata, Gigaspora rosea and Rhizophagus clarus) and a total control (without fungus and bacteria), with three replicates per treatment, totaling 60 experimental units. The height, diameter, number of leaves, fresh root matter, shoot dry matter, $\mathrm{N}$ and $\mathrm{P}$ content and accumulation, intensity and percentage of root colonization, active and total extrarradicular mycelium and the number of spores were evaluated after 12 months. There were differentiated results of treatments for each cultivar, showing that for some attributes, there was an isolated effect of FMA or co-inoculation of FMA and rhizobacteria, which provided significant increases on the growth and development of olive tree seedlings.
\end{abstract}

Keywords: Growth-promoting bacteria; Microbiological inputs; Mycorrhiza

\footnotetext{
I Bióloga, MSc., Instituto de Recursos Naturais, Universidade Federal de Itajubá, Av. BPS, 1303, CEP 37500-903, Itajubá (MG), Brasil. samaramlopesc@gmail.com (ORCID: 0000-0002-6643-6478)

II Engenheiro Agrônomo, Dr., Professor do Instituto de Recursos Naturais, Universidade Federal de Itajubá, Av. BPS, 1303, CEP 37500-903, Itajubá (MG), Brasil. rogerio.melloni@gmail.com (ORCID: 0000-0002-2724-2603)
} 


\section{Introdução}

A oliveira (Olea europaea L.) é uma planta de porte arbóreo que pertence à família Oleaceae, produz frutos comestíveis e apresenta um ciclo de vida perene, destacando-se por sua longevidade (COUTINHO et al., 2015). Os frutos são denominados azeitonas, sendo estes consumidos in natura, como as azeitonas de mesa, ou usados para extração do azeite que, assim como as azeitonas de mesa, são consumidos mundialmente.

No Brasil, tal cultura se estabeleceu no início do século XIX, em quase todos os estados, porém, com maior expressividade nas regiões Sudeste e Sul (Minas Gerais, Rio de Janeiro, São Paulo, Paraná, Santa Catarina e Rio Grande do Sul) (VIEIRA NETO et al., 2008). Entretanto, o país não possui plantações comerciais suficientes para atender às demandas comerciais do mercado interno, o que faz com que importe todo o azeite de oliva e azeitona que consome, principalmente de países como Portugal, Espanha, Itália, Grécia e países sul-americanos como Argentina e Chile (BALLUS et al., 2014).

As regiões altas da Serra da Mantiqueira, no Brasil, já demonstram viabilidade para produção dessa cultura, conforme demonstrado nos estudos de Oliveira et al. (2012) e Ballus et al. (2014), ainda que não possuam as mesmas condições climáticas que os países do mediterrâneo. Diversos fatores contribuem para o sucesso da produtividade dessa cultura, que vão desde os fatores climáticos até o manejo adequado da espécie. Neste contexto, o uso de tecnologias microbiológicas na produção de mudas de oliveiras, em especial aquelas envolvendo grupos funcionais como os fungos micorrízicos arbusculares (FMA) e as rizobactérias pode ser uma alternativa e ferramenta de gestão ambiental.

Os FMAs desempenham papéis essenciais na natureza, apresentando estruturas denominadas hifas, que atuam como uma extensão do sistema radicular das plantas e são de extrema importância para absorção efetiva de nutrientes, principalmente o fósforo, e água pelas plantas, além de atuar na estruturação do solo, na tolerância a estresses abióticos e resistência a doenças em plantas (SMITH; READ, 2008; CARDOSO; ANDREOTE, 2016). Para a cultura da oliveira, existem diversos trabalhos, com resultados positivos relacionados à utilização de FMAs, como os de Bompadre et al. (2014) na Argentina, Montes-Borrego, Metsis e Landa (2014) na Espanha, Bati, Santilli e Lombardo (2015) na Itália, Malik, Nuñez e McKeever (2017) nos Estados Unidos, Mechri et al. (2014), Tekaya et al. (2017) na Tunísia, e de Meddad-Hamza et al. (2017) na Argélia. No Brasil, os trabalhos de Vieira, Melloni e Vieira Neto (2011) e Ferreira et al. (2015) foram conduzidos e evidenciaram a formação de associações micorrízicas em condição natural, e o efeito positivo dos microrganismos no crescimento das mudas, respectivamente.

$\mathrm{O}$ uso de insumos à base de rizobactérias para a melhoria do crescimento e desenvolvimento de plantas também tem sido estudado em diversas culturas (CARVALHO et al., 2009; PAIS et al., 2016). As rizobactérias promotoras do crescimento em plantas (RBPC), por exemplo, proporcionam às plantas um melhor desenvolvimento, por meio de diferentes mecanismos, como suplementação de nutrientes não disponíveis ou inibição do desenvolvimento de pragas e patógenos de plantas, produção de bactericidas, antifúngicos, hormônios vegetais tais como auxinas, citocininas, giberelinas, etileno e ácido abscísico (VEJAN et al., 2016). No entanto, especificamente para oliveira, há poucos trabalhos, com resultados positivos, como os de Peyvandi et al. (2010), no Irã e Montero-Calasanz et al. (2013), na Espanha. No Brasil, apenas o trabalho de Silva et al. (2017) avaliou o efeito de rizobactérias diazotróficas não simbióticas no enraizamento de estacas semilenhosas de oliveira, confirmando-se seu potencial de utilização em virtude da produção de ácido indolacético.

Apesar do efeito benéfico da coinoculação de FMAs com rizobactérias no crescimento e desenvolvimento de diferentes culturas, como o milho (Zea mays var. rugosa) (DHAWI; DATTA; RAMAKRISHNA, 2015) e tomateiro (Solanum lycopersicum L.) (BONA et al., 2016), são inexistentes trabalhos com coinoculação de FMAs e rizobactérias na cultura da oliveira (Olea europaea L.). No entanto, sabe-se que há uma potencialização de efeitos benéficos às plantas quando se inoculam ambos os simbiontes, em função do aumento da disponibilidade e absorção de nutrientes, tolerância a estresses bióticos e abióticos, e proteção contra patógenos (RAMASAMY et al., 2011). Assim, o objetivo do presente estudo foi analisar o efeito da coinoculação de FMAs e rizobactérias no crescimento de mudas de duas importantes cultivares de oliveira no Brasil, Arbequina e Maria da Fé. 


\section{Material e métodos}

Dois experimentos foram instalados em condições controladas de casa de vegetação da Universidade Federal de Itajubá (Unifei), em Itajubá - MG, um para cada cultivar de oliveira (Arbequina e Maria da Fé) em Delineamento Inteiramente Casualizado (DIC) e esquema fatorial $(3 \times 3+1)$, sendo 3 isolados de rizobactérias identificados respectivamente pelo Laboratório de Biotecnologia do Solo da Embrapa Soja, como Pseudomonas sp, Paenibacillus sp1 e Paenibacillus sp2, combinados com 3 espécies de FMAs (Acaulospora scrobiculata, Gigaspora rosea e Rhizophagus clarus) e um controle total (sem fungo e sem bactéria), com três repetições por tratamento, totalizando 60 unidades experimentais. As cultivares de oliveira, as espécies de rizobactérias e de FMAs selecionados para o estudo foram definidos em função dos resultados positivos de Ferreira et al. (2015) e Silva et al. (2017), quando inoculados isoladamente, respectivamente.

Cada unidade experimental foi composta por uma estaca semilenhosa de oliveira de $10 \mathrm{~cm}$, com 2 pares de folhas, previamente enraizada em ácido indolbutírico (AIB) a $3 \mathrm{~g} \mathrm{~L}^{-1}$ por $5 \mathrm{~s}$, em um saco de polietileno com capacidade $1,5 \mathrm{~kg}$. O substrato desses sacos era composto por areia lavada e esterilizada em autoclave $\left(1 \mathrm{~h} \mathrm{a} 121^{\circ} \mathrm{C}\right.$, duas vezes, em dias alternados) e duas partes de terra (Latossolo Vermelho Amarelo, distrófico, textura média). Cada unidade experimental recebeu uma suspensão de rizobactérias, conforme metodologia proposta por Silva et al. (2017), enquanto que 100 esporos de cada espécie de FMAs foram aplicados por planta ao lado das raízes próximas ao colo.

O crescimento das mudas foi acompanhado por 12 meses e avaliado por meio dos atributos altura, diâmetro, número de folhas, matéria fresca de raiz, matéria seca da parte aérea (após secagem em estufa por $60^{\circ} \mathrm{C}$, durante 72 horas), teor e acúmulo de $\mathrm{N}$ e $\mathrm{P}$ da parte aérea, intensidade e porcentagem colonização radicular (PHILLIPS; HAYMAN, 1970; GIOVANNETTI; MOSSE, 1980), micélio extrarradicular ativo e total (MELLONI; CARDOSO, 1999) e número de esporos (GERDEMANN; NICOLSON, 1963).

Para cada atributo, realizou-se o teste de Shapiro Wilk a $5 \%$ de probabilidade para verificar o pressuposto de normalidade. Para os dados que não seguiram esse pressuposto, foi aplicada a transformação $\sqrt{ } \mathrm{x}+1$. Posteriormente, realizou-se a análise de variância (ANOVA) para verificar se houve diferença entre as fontes de variação (níveis do fator rizobactérias e FMAs). Para os atributos que apresentaram significância, realizou-se a comparação de médias pelo teste Skott e Knott a 5\% de probabilidade. Para estas análises, foi utilizado o programa estatístico R, com o pacote estatístico ExpDes.pt (FERREIRA; CAVALCANTI; NOGUEIRA, 2011).

\section{Resultados e discussão}

\section{Cultivar Arbequina}

Os atributos altura, diâmetro do colo, matéria fresca de raiz, matéria seca da parte aérea, teor e acúmulo de fósforo e micélio extrarradicular total apresentaram efeito da coinoculação de FMAs e rizobactérias (Tabela 1).

Esses resultados corroboram os encontrados por Kumar et al. (2015), os quais mostraram que a inoculação com todas as rizobactérias e FMAs promoveu aproximadamente o dobro de matéria seca da parte aérea, raiz e altura de planta, quando comparada ao controle, resultando em maior produtividade. Segundo Kumar et al. (2015), esse aumento pode ter sido devido à capacidade das rizobactérias aumentarem o efeito micorrízico, uma vez que os parâmetros físico químicos (liberação de exsudatos radiculares, acidificação pela liberação de prótons, alterações de $\mathrm{pH}$ do solo, secreção de fitormônios pelas rizobactérias, entre outros) são regulados por essa interação e, consequentemente, afetam o crescimento da planta, conclusões estas que podem ser replicadas para essa cultivar de oliveira.

Desses atributos anteriormente citados, em que houve efeito da coinoculação entre FMAs e rizobactérias, os atributos altura, diâmetro, matéria fresca de raiz, teor de $\mathrm{P}$ e micélio extrarradicular total não apresentaram diferença estatística entre o fatorial e o controle (Tabela 1), diferentemente daquele observado para os atributos matéria seca da parte aérea e acúmulo de $\mathrm{P}$, em que o fatorial diferiu estatisti- 
camente do controle. Esses resultados corroboram aqueles encontrados nos trabalhos de Rodríguez-Romero, Guerra, Jaizme-Vega (2005), que ao estudarem os efeitos do FMA Glomus manihotis e um consórcio de rizobactérias Bacillus spp. sobre o crescimento e nutrição de bananeira durante a fase de formação das mudas, verificaram um aumento significativo na matéria fresca de raiz, matéria seca da parte aérea e altura, significativamente maiores que o controle, sem inoculação. Os autores salientam os benefícios da dupla inoculação proporcionados às mudas de bananeira (Musa acuminata) como resultados da alta especificidade em termos de compatibilidade funcional envolvida nas interações microbianas da rizosfera. Um maior peso de matéria seca total foi observada no trabalho de Kim et al. (2010), com um experimento que avaliou o crescimento de pimenta-vermelha (Capsicum annuum L.) inoculada com duas cepas de $M e-$ thylobacterium oryzae e um consórcio de três espécies de FMAs (Acaulospora longula, Glomus clarum e Glomus intraradiaces). Tais autores verificaram que a coinoculação promoveu maior matéria seca da parte aérea das mudas de pimenta que a inoculação separada, quando comparada ao controle, sem inoculação. Resultados semelhantes foram encontrados por Kumar et al. (2015), ao estudarem a interação entre FMAs e rizobactérias para acultura do milho (Zea mays).

Quanto ao acúmulo de fósforo (Tabela 1), o tratamento fatorial proporcionou maiores incrementos de fósforo $\left(3,3 \mathrm{~g} \mathrm{planta}^{-1}\right)$ do que o controle $\left(0,6 \mathrm{~g} \mathrm{planta}^{-1}\right)$, diferindo estatisticamente deste. Já acerca da coinoculação, as combinações dos FMAs Acaulospora scrobiculata + Paenibacillus sp1 (9,8 g planta $\left.^{-1}\right)$ e Acaulospora scrobiculata + Paenibacillus sp2 $\left(9,0\right.$ g planta $\left.^{-1}\right)$ promoveram os maiores acúmulos de fósforo, não diferindo estatisticamente entre si. No trabalho de Kim et al. (2010), anteriormente citado, verificou-se também um maior acúmulo de nutrientes (todos os macro e micronutrientes) nas raízes e parte aérea de plantas de pimenta coinoculadas.

Tabela 1- Efeito da coinoculação de FMAs e rizobactérias na cultivar de oliveira Arbequina.

Table 1 - Effect of co-inoculation of AMF and rhizobacteria in Arbequina cultivar.

\begin{tabular}{|c|c|c|c|c|c|}
\hline \multicolumn{6}{|c|}{ Altura (cm) } \\
\hline & Pseudomonas sp & Paenibacillus sp1 & Paenibacillus sp2 & Fatorial & Controle \\
\hline Rhizophagus clarus & $24,5 \mathrm{Ab}$ & $23,7 \mathrm{Aa}$ & $15,3 \mathrm{Bb}$ & & \\
\hline Acaulospora scrobiculata & $33,2 \mathrm{Aa}$ & $31,3 \mathrm{Aa}$ & $39,0 \mathrm{Aa}$ & $26,3 \mathrm{~A}$ & $24,7 \mathrm{~A}$ \\
\hline Gigaspora rosea & $21,0 \mathrm{Bb}$ & $29,3 \mathrm{Aa}$ & $19,2 \mathrm{Bb}$ & & \\
\hline \multicolumn{6}{|c|}{ Diâmetro (cm) } \\
\hline & Pseudomonas sp & Paenibacillus sp1 & Paenibacillus sp2 & Fatorial & Controle \\
\hline Rhizophagus clarus & $2,9 \mathrm{Bb}$ & $4,3 \mathrm{Aa}$ & $2,4 \mathrm{Bb}$ & & \\
\hline Acaulospora scrobiculata & 3,9 Aa & $2,9 \mathrm{Bb}$ & $4,3 \mathrm{Aa}$ & $3,2 \mathrm{~A}$ & $3,0 \mathrm{~A}$ \\
\hline Gigaspora rosea & $2,4 \mathrm{Ab}$ & $2,7 \mathrm{Ab}$ & $2,6 \mathrm{Ab}$ & & \\
\hline \multicolumn{6}{|c|}{ Matéria Fresca de raiz (g planta $\left.{ }^{-1}\right)$} \\
\hline & Pseudomonas sp & Paenibacillus sp1 & Paenibacillus sp2 & Fatorial & Controle \\
\hline Rhizophagus clarus & $0,8 \mathrm{Aa}$ & $0,4 \mathrm{Aa}$ & $1,1 \mathrm{Aa}$ & & \\
\hline Acaulospora scrobiculata & $1,4 \mathrm{Aa}$ & $0,8 \mathrm{Aa}$ & $1,6 \mathrm{Aa}$ & $0,9 \mathrm{~A}$ & $0,5 \mathrm{~A}$ \\
\hline Gigaspora rosea & $0,5 \mathrm{Ba}$ & $1,2 \mathrm{Aa}$ & $0,2 \mathrm{Bb}$ & & \\
\hline \multicolumn{6}{|c|}{ Matéria seca parte aérea (g planta-1) } \\
\hline & Pseudomonas sp & Paenibacillus sp1 & Paenibacillus sp2 & Fatorial & Controle \\
\hline Rhizophagus clarus & $2,4 \mathrm{Ab}$ & $2,5 \mathrm{Aa}$ & $0,3 \mathrm{Bb}$ & & \\
\hline Acaulospora scrobiculata & 4,9 Aa & $2,4 \mathrm{Ba}$ & $5,8 \mathrm{Aa}$ & $2,4 \mathrm{~A}$ & $0,9 \mathrm{~B}$ \\
\hline Gigaspora rosea & $1,2 \mathrm{Ab}$ & $1,3 \mathrm{Aa}$ & $1,0 \mathrm{Ab}$ & & \\
\hline
\end{tabular}

Continua... 
Tabela 1 - Conclusão...

Table 1 - Conclusion...

\begin{tabular}{|c|c|c|c|c|c|}
\hline \multicolumn{6}{|c|}{ Teor de $P\left(\mathrm{~g} \mathrm{~kg}^{-1}\right)$} \\
\hline & Pseudomonas sp & Paenibacillus sp1 & Paenibacillus sp2 & Fatorial & Controle \\
\hline Rhizophagus clarus & $1,2 \mathrm{Aa}$ & $0,4 \mathrm{Ab}$ & $1,2 \mathrm{Aa}$ & & \\
\hline Acaulospora scrobiculata & $1,7 \mathrm{Ba}$ & $2,7 \mathrm{Aa}$ & $1,5 \mathrm{Ba}$ & $1,1 \mathrm{~A}$ & $0,7 \mathrm{~A}$ \\
\hline Gigaspora rosea & $0,5 \mathrm{Aa}$ & $0,6 \mathrm{Ab}$ & $0,7 \mathrm{Aa}$ & & \\
\hline \multicolumn{6}{|c|}{ Acúmulo de $\mathbf{P}$ (g planta $\left.{ }^{-1}\right)$} \\
\hline & Pseudomonas sp & Paenibacillus sp1 & Paenibacillus sp2 & Fatorial & Controle \\
\hline Rhizophagus clarus & $1,1 \mathrm{Ab}$ & $0,9 \mathrm{Ab}$ & $0,4 \mathrm{Ab}$ & & \\
\hline Acaulospora scrobiculata & $6,3 \mathrm{Ba}$ & $9,8 \mathrm{Aa}$ & $9,0 \mathrm{Aa}$ & $3,3 \mathrm{~A}$ & $0,6 \mathrm{~B}$ \\
\hline Gigaspora rosea & $0,5 \mathrm{Ab}$ & $1,0 \mathrm{Ab}$ & $0,7 \mathrm{Ab}$ & & \\
\hline \multicolumn{6}{|c|}{ Micélio extrarradicular total ( $\mathrm{m} \mathrm{g}^{-1}$ solo seco) } \\
\hline & Pseudomonas sp & Paenibacillus sp1 & Paenibacillus sp2 & Fatorial & Controle \\
\hline Rhizophagus clarus & $0,7 \mathrm{Aa}$ & $1,5 \mathrm{Aa}$ & $1,5 \mathrm{Ab}$ & & \\
\hline Acaulospora scrobiculata & $1,1 \mathrm{Aa}$ & $1,0 \mathrm{Aa}$ & $0,6 \mathrm{Ac}$ & $1,2 \mathrm{~A}$ & $1,0 \mathrm{~A}$ \\
\hline Gigaspora rosea & $1,1 \mathrm{Ba}$ & $1,2 \mathrm{Ba}$ & $2,4 \mathrm{Aa}$ & & \\
\hline
\end{tabular}

Médias seguidas de letras iguais maiúsculas nas linhas e minúsculas nas colunas não diferem entre si pelo teste de Skott e Knott a 5\% de probabilidade.

Para os atributos microbiológicos, verificou-se que para micélio extrarradicular total (Tabela 1), a combinação do FMA Gigaspora rosea + Paenibacillus sp2 promoveu maior comprimento do micélio extrarradicular total $\left(2,4 \mathrm{~m} \mathrm{~g}^{-1}\right)$, em relação às demais combinações. Em relação ao micélio extrarradicular ativo, não houve interação significativa entre os tratamentos. Valadares, Mescolotti e Cardoso (2016) afirmaram que durante a colonização micorrízica pelo fungo, o grande volume de micélio é capaz de explorar regiões nas quais as raízes das plantas não conseguem alcançar, absorvendo nutrientes essências às plantas, como fósforo, e também otimizando o uso da água e a estabilidade dos agregados do solo. Para o presente estudo com oliveira, a quantidade produzida de micélio nos tratamentos não diferiu estatisticamente do controle, no qual a presença de fungos não micorrízicos (hifas septadas) também foi evidenciada. No entanto, pode-se verificar que a quantidade de micélio do controle não foi suficiente para aumentar o acúmulo de P na planta (Tabela 1) e nem promover formação de massa seca da parte aérea (Tabela 1), como observado para os tratamentos de coinoculação, com efeitos positivos.

Não houve efeito da interação de FMAs e rizobactérias para número de folhas, mas somente o efeito dos FMAs isoladamente. O mesmo se deu para teor e acúmulo de nitrogênio, intensidade e porcentagem de colonização radicular e número de esporos (Tabela 2).

Tabela 2 - Efeito de FMAs no crescimento e formação de micorriza na cultivar de oliveira Arbequina.

Table 2 - Effect of AMF on the growth and mycorriza formation in Arbequina cultivar.

\begin{tabular}{|c|c|c|c|c|c|}
\hline & $\begin{array}{c}\text { Rhizophagus } \\
\text { clarus }\end{array}$ & $\begin{array}{l}\text { Acaulospora } \\
\text { scrobiculata }\end{array}$ & $\begin{array}{l}\text { Gigaspora } \\
\text { rosea }\end{array}$ & Fatorial & Controle \\
\hline Número de folhas $\left(\mathrm{g}_{\text {planta }}{ }^{-1}\right)$ & $24,4 \mathrm{~B}$ & $54,8 \mathrm{~A}$ & $13,1 \mathrm{~B}$ & $30,8 \mathrm{~A}$ & $20,0 \mathrm{~A}$ \\
\hline Teor de $\mathrm{N}\left(\mathrm{g} \mathrm{kg}^{-1}\right)$ & $12,5 \mathrm{~B}$ & $15,4 \mathrm{~A}$ & $13,6 \mathrm{~B}$ & $13,9 \mathrm{~A}$ & $14,4 \mathrm{~A}$ \\
\hline Acúmulo de $\mathrm{N}\left(\right.$ g planta $\left.^{-1}\right)$ & $19,4 \mathrm{~B}$ & $71,1 \mathrm{~A}$ & $16,1 \mathrm{~B}$ & $35,6 \mathrm{~A}$ & $37,0 \mathrm{~A}$ \\
\hline Intensidade de Colonização Radicular (\%) & $1,8 \mathrm{~B}$ & $22,6 \mathrm{~A}$ & $0,0 \mathrm{~B}$ & $8,1 \mathrm{~A}$ & $3,0 \mathrm{~A}$ \\
\hline Porcentagem de Colonização Radicular (\%) & $2,3 \mathrm{~B}$ & $55,0 \mathrm{~A}$ & $4,0 \mathrm{~B}$ & $20,4 \mathrm{~A}$ & $17,3 \mathrm{~A}$ \\
\hline Número de Esporos (n⿳o $50 \mathrm{~mL}^{-1}$ ) & $2,3 \mathrm{~B}$ & $6,3 \mathrm{~B}$ & $10,8 \mathrm{~A}$ & $6,5 \mathrm{~A}$ & $2,3 \mathrm{~A}$ \\
\hline
\end{tabular}

Médias seguidas de letras iguais na linha não diferem entre si pelo teste de Skott e Knott a 5\% de probabilidade. 
Esses resultados corroboram os encontrados por Lima et al. (2011), em mudas de mamoeiro (Carica papaya L.), avaliando a coinoculação de bactérias diazotróficas (Stenotrophomonas maltophilia e Azospirillum sp) e FMAs (Glomus clarum + Gigaspora margarita). Os autores verificaram que apenas os FMAs em estudo foram responsivos às variáveis de crescimento e desenvolvimento, independentemente da presença da bactéria. Segundos esses autores, possivelmente pode haver uma competição entre microrganismos quando coinoculados em solo com baixa disponibilidade de $\mathrm{P}$, o que interferiria no desenvolvimento das plantas.

Para todos os atributos citados (número de folhas, teor e acúmulo de nitrogênio, intensidade e porcentagem de colonização radicular e número de esporos), em que houve apenas efeito isolado do FMA, não houve diferença estatística entre o fatorial e o controle, sem inoculação. Quanto ao efeito isolado do FMA, para todos esses atributos destacou-se a inoculação com Acaulospora scrobiculata, independentemente da presença das rizobactérias, exceto para o número de esporos, em que o FMA G. rosea proporcionou maior número de esporos e diferiu estatisticamente dos demais FMAs (Tabela 2).

Apesar de não ter havido efeito da coinoculação para a porcentagem de colonização neste estudo, o efeito do FMA sobre tal atributo pode ser observado em outros trabalhos realizados como de Meddad Hamza et al. (2010), que, ao avaliarem o potencial de colonização de Glomus mossae e Glomus intraradices sobre o crescimento de cultivares de oliveira Aglandou micropropagadas, verificaram colonização de $73,9 \%$ e $67,3 \%$, respectivamente. Esse resultado evidencia alta afinidade destes fungos com a cultivar, já que sua inoculação promoveu melhor crescimento das mudas, verificado nos atributos analisados, quando comparado com o controle, sem inoculação. Calvente et al. (2004), ao analisarem a diversidade natural de FMA na rizosfera de cultivares de oliveiras (Olea europaea L.) Arbequina e Leccino e avaliarem a eficácia dos isolados de FMA nativos para atuarem como inoculantes para essas cultivares, também observaram que os FMAs Glomus mossae e Glomus intraradices proporcionaram alta porcentagem de colonização, de $77 \%$ e $72 \%$, respectivamente na cultivar Arbequina, resultados semelhantes aos encontrados neste trabalho (55\%), para o FMA Acaulospora scrobiculata.

Em relação aos efeitos dos FMAs sobre o desenvolvimento de diversas culturas Seifi et al. (2014) investigaram o efeito da inoculação micorrízica utilizando duas espécies de FMAs (Glomus mosseae e Glomus intraradices) sobre atributos de crescimento e fitoquímicos de cultivares de oliveira Koroneiki e Valanolia, no Irã. Os resultados foram diferenciados para as cultivares em relação aos atributos, com efeitos positivos da inoculação micorrízica em todos os atributos. Os autores afirmaram que a inoculação de FMA em oliveiras, na fase de viveiro, pode ser considerada uma forma simples e efetiva de aumentar o crescimento das mudas, permitindo a produção de plantas saudáveis e vigorosas. Esse resultado pode ser replicado aos obtidos no presente trabalho, uma vez que quando do efeito do FMA, os atributos número de folhas, teor e acúmulo de nitrogênio, intensidade e porcentagem de colonização radicular apresentaram valores maiores estatisticamente com a utilização do FMA Acaulospora scrobiculata.

No geral, as coinoculações beneficiaram o desenvolvimento das mudas das cultivares de oliveira Arbequina, com incrementos na altura, diâmetro, matéria fresca de raiz, matéria seca da parte aérea, e estado nutricional das mudas com maior absorção de $\mathrm{P}$, mostrando o seu potencial de utilização na produção de mudas dessa cultivar.

\section{Cultivar Maria da Fé}

Houve resultado diferenciado dos tratamentos para cada um dos atributos analisados para a cultivar Maria da Fé. No entanto, para número de folhas, teor de nitrogênio e micélio extrarradicular ativo não houve diferença estatística entre o tratamento fatorial (tratamentos coinoculados) e o controle, enquanto as coinoculações em todos esses atributos, proporcionaram efeitos diversificados (Tabela 3), assim como observado para a cultivar Arbequina. Resultados em que houve efeito da coinoculação sobre os atributos de crescimento também foram encontrados por Aguirre-Medina e Moreno (2016), ao estudarem o efeito da inoculação isolada ou combinada do FMA Rhizophagus intraradices com as rizobactérias Pseudomonas fluorescens e Azospirillum brasilense, sobre o crescimento, produtividade e colonização de pimenta (Capsicum annuum L.). Dependendo da combinação, tais autores encontraram frutos maiores de pimenta 
ou maior número de frutos, resultados associados à capacidade de colonização e interação de microrganismos com a planta.

Quanto à coinoculação, para número de folhas, as combinações do FMA Acaulospora scrobiculata + Paenibacillus sp2 (91,3) e Acaulospora scrobiculata + Pseudomonas sp $(80,3)$ não diferiram estatisticamente entre si e promoveram os maiores valores desse atributo comparadas às demais (Tabela 3 ). Já para o teor de nitrogênio (Tabela 3B), as combinações do FMA Gigaspora rosea + Paenibacillus sp1 (11,2 $\left.\mathrm{g} \mathrm{kg}^{-1}\right)$, Acaulospora scrobiculata + Paenibacillus $\mathrm{sp} 2\left(10,8 \mathrm{~g} \mathrm{~kg}^{-1}\right)$ e Rhizophagus clarus + Pseudomonas $\mathrm{sp}\left(10,8 \mathrm{~g} \mathrm{Kg}^{-1}\right)$ proporcionaram os maiores valores e diferiram dos demais tratamentos.

Tabela 3- Efeito da coinoculação de FMAs e rizobactérias na cultivar de oliveira Maria da Fé.

Table 3 - Effect of co-inoculation of AMF and rhizobacteria in the 'Maria da Fé' cultivar.

\begin{tabular}{|c|c|c|c|c|c|}
\hline \multicolumn{6}{|c|}{ Número de folhas (g planta ${ }^{-1}$ ) } \\
\hline & Pseudomonas sp & Paenibacillussp1 & Paenibacillus sp2 & Fatorial & Controle \\
\hline Rhizophagus clarus & $10,7 \mathrm{Ab}$ & $29,0 \mathrm{Aa}$ & $8,3 \mathrm{Ab}$ & & \\
\hline Acaulospora scrobiculata & $80,3 \mathrm{Aa}$ & $38,0 \mathrm{Ba}$ & $91,3 \mathrm{Aa}$ & $35,7 \mathrm{~A}$ & $17,3 \mathrm{~A}$ \\
\hline Gigaspora rosea & $27,0 \mathrm{Ab}$ & $8,0 \mathrm{Aa}$ & $28,7 \mathrm{Ab}$ & & \\
\hline \multicolumn{6}{|c|}{ Teor de N $\left(\mathrm{g} \mathrm{kg}^{-1}\right)$} \\
\hline & Pseudomonas sp & Paenibacillus sp1 & Paenibacillus sp2 & Fatorial & Controle \\
\hline Rhizophagus clarus & $10,8 \mathrm{Aa}$ & $9,5 \mathrm{Ab}$ & $7,2 \mathrm{Bb}$ & & \\
\hline Acaulospora scrobiculata & $9,4 \mathrm{Ab}$ & $7,1 \mathrm{Bc}$ & $10,8 \mathrm{Aa}$ & $9,3 \mathrm{~A}$ & $8,8 \mathrm{~A}$ \\
\hline Gigaspora rosea & $8,6 \mathrm{Bb}$ & $11,2 \mathrm{Aa}$ & $9,4 \mathrm{Ba}$ & & \\
\hline \multicolumn{6}{|c|}{ Acúmulo de $\mathbf{N}$ (g planta $\left.{ }^{-1}\right)$} \\
\hline & Pseudomonas sp & Paenibacillus sp1 & Paenibacillus sp2 & Fatorial & Controle \\
\hline Rhizophagus clarus & $13,6 \mathrm{Ab}$ & $14,6 \mathrm{Ab}$ & $10,0 \mathrm{Ab}$ & & \\
\hline Acaulospora scrobiculata & $44,9 \mathrm{Ba}$ & $42,2 \mathrm{Ba}$ & $95,6 \mathrm{Aa}$ & $32,4 \mathrm{~A}$ & $17,2 \mathrm{~B}$ \\
\hline Gigaspora rosea & $35,4 \mathrm{Aa}$ & $11,9 \mathrm{Ab}$ & $23,0 \mathrm{Ab}$ & & \\
\hline \multicolumn{6}{|c|}{ Porcentagem de Colonização Radicular } \\
\hline & Pseudomonas sp & Paenibacillus sp1 & Paenibacillus sp2 & Fatorial & Controle \\
\hline Rhizophagus clarus & $13,2 \mathrm{Aa}$ & $5,0 \mathrm{Ab}$ & $5,3 \mathrm{Ab}$ & & \\
\hline Acaulospora scrobiculata & $12,1 \mathrm{Ba}$ & $15,1 \mathrm{Ba}$ & $50,1 \mathrm{Aa}$ & $12,8 \mathrm{~A}$ & $4,3 \mathrm{~B}$ \\
\hline Gigaspora rosea & $5,7 \mathrm{Aa}$ & $0,0 \mathrm{Bc}$ & $8,7 \mathrm{Ab}$ & & \\
\hline \multicolumn{6}{|c|}{ Micélio extrarradicular ativo ( $\mathrm{m} \mathrm{g}^{-1}$ solo seco) } \\
\hline & Pseudomonas sp & Paenibacillus sp1 & Paenibacillus sp2 & Fatorial & Controle \\
\hline Rhizophagus clarus & $0,02 \mathrm{Ab}$ & $0,02 \mathrm{Aa}$ & $0,00 \mathrm{Aa}$ & & \\
\hline Acaulospora scrobiculata & $0,00 \mathrm{Ab}$ & $0,02 \mathrm{Aa}$ & $0,01 \mathrm{Aa}$ & $0,01 \mathrm{~A}$ & $0,02 \mathrm{~A}$ \\
\hline Gigaspora rosea & 0,07Aa & $0,00 \mathrm{Ba}$ & $0,00 \mathrm{Ba}$ & & \\
\hline
\end{tabular}

Micélio extrarradicular total $\left(\mathrm{m} \mathrm{g}^{-1}\right.$ solo seco)

\begin{tabular}{lccccc}
\hline & Pseudomonas sp & Paenibacillus sp1 & Paenibacillus sp2 & Fatorial & Controle \\
\hline Rhizophagus clarus & $0,7 \mathrm{Ba}$ & $0,5 \mathrm{Bb}$ & $2,9 \mathrm{Aa}$ & & \\
Acaulospora scrobiculata & $0,7 \mathrm{Ba}$ & $2,2 \mathrm{Aa}$ & $1,3 \mathrm{Bb}$ & $1,2 \mathrm{~B}$ & $2,3 \mathrm{~A}$ \\
Gigaspora rosea & $1,4 \mathrm{Aa}$ & $0,4 \mathrm{Bb}$ & $0,7 \mathrm{Bb}$ & \\
\hline
\end{tabular}

Médias seguidas de letras iguais maiúsculas nas linhas e minúsculas nas colunas não diferem entre si pelo teste de Skott e Knott a 5\% de probabilidade. 
O acúmulo de nitrogênio apresentou diferença estatística entre o tratamento fatorial e o controle, no qual o primeiro apresentou maior acúmulo de nitrogênio $\left(32,4 \mathrm{~g}_{\text {planta }}{ }^{-1}\right)$ do que o controle $(17,2 \mathrm{~g}$ planta $^{-1}$ ), diferindo estatisticamente deste (Tabela 3). Quanto à coinoculação, a combinação do FMA Acaulospora scrobiculata + Paenibacillus sp2 $\left(95,6 \mathrm{~g} \mathrm{planta}^{-1}\right)$ proporcionou o maior incremento de nitrogênio, diferindo estatisticamente das demais combinações.

Para a porcentagem de colonização radicular, o tratamento fatorial apresentou maior porcentagem de colonização $(12,8 \%)$ do que o controle (4,3\%), diferindo estatisticamente deste (Tabela 3$)$. No que diz respeito à coinoculação, a combinação do FMA Acaulospora scrobiculata + Paenibacillus sp2 (50,1\%) proporcionou uma maior porcentagem de colonização, diferindo estatisticamente das demais combinações (Tabela 3). Similarmente a esses resultados, Oliveira et al. (2010), ao trabalharem com inoculação isolada e combinada dos FMAs Glomus entunicatum e a mistura de Glomus etunicatum com Gigaspora margarita com as rizobactérias Bacillus thuringiensis e Bacillus pumilus em plantas de gengibre (Zingiber spectabile Griff), verificaram que a combinação Bacillus pumilus + Glomus entunicatum/Gigaspora margarita favoreceu a colonização micorrízica no gengibre, com valores médios de 60,3\%. Esses autores justificaram que tal fato pode estar relacionado à capacidade de algumas rizobactérias estimularem o crescimento da biomassa do micélio, propiciando a colonização radicular pelas hifas fúngicas, o que também pode ter acontecido para a cultivar de oliveira Maria da Fé em estudo.

Para o micélio extrarradicular ativo, não houve diferença estatística entre o tratamento fatorial $\left(0,01 \mathrm{~m} \mathrm{~g}^{-1}\right)$ e o controle $\left(0,02 \mathrm{~m} \mathrm{~g}^{-1}\right)$, mostrando que outros fungos não micorrízicos estiveram presentes nas plantas não inoculadas. Com relação ao efeito da coinoculação, a combinação do FMA Gigaspora rosea + Pseudomonas sp $\left(0,07 \mathrm{~m} \mathrm{~g}^{-1}\right)$ proporcionou os maiores comprimentos de micélio ativo, diferindo estatisticamente das demais combinações.

O micélio extrarradicular total apresentou diferença estatística entre o tratamento fatorial e o controle, no qual o controle $\left(2,3 \mathrm{~m} \mathrm{~g}^{-1}\right)$ apresentou valor estatisticamente superior ao fatorial $\left(1,2 \mathrm{~m} \mathrm{~g}^{-1}\right)$. No entanto, esse micélio não é de FMAs e não esteve ligado ao crescimento ou desenvolvimento das plantas de oliveira. Tal micélio foi originado, possivelmente, de contaminação aérea, comum em estudos dessa natureza (WEBER; AMORIM, 1994; COSTA et al., 2001). Diferentemente, a combinação do FMA Rhizophagus clarus + Paenibacillus sp2 (2,9 $\left.\mathrm{m} \mathrm{g}^{-1}\right)$ e Acaulospora scrobiculata + Paenibacillus sp1 (2,2 m $\left.\mathrm{g}^{-1}\right)$ promoveu os maiores comprimentos de micélio e não diferiu estatisticamente entre si.

Não houve efeito da interação entre FMAs e rizobactérias para a altura, mas apenas efeito para o FMA isoladamente, com destaque para Acaulospora scrobiculata. O mesmo se deu para diâmetro do colo, matéria seca da parte aérea, teor e acúmulo de fósforo e intensidade de colonização radicular (Tabela 4). Nos trabalhos de Ferreira et al. (2015), esta mesma espécie de fungo também se destacou como eficiente na cultivar de oliveira Maria da Fé, principalmente pela maior produção de matéria seca das raízes.

Tabela 4 - Efeito dos FMAs na cultivar de oliveira Maria da Fé.

Table 4 - Effect of AMF in the 'Maria da Fé' cultivar.

\begin{tabular}{|c|c|c|c|c|c|}
\hline & $\begin{array}{c}\text { Rhizophagus } \\
\text { clarus }\end{array}$ & $\begin{array}{l}\text { Acaulospora } \\
\text { scrobiculata }\end{array}$ & $\begin{array}{l}\text { Gigaspora } \\
\text { rósea }\end{array}$ & Fatorial & Controle \\
\hline Altura $(\mathrm{cm})$ & $19,8 \mathrm{~B}$ & $35,9 \mathrm{~A}$ & $21,2 \mathrm{~B}$ & $25,6 \mathrm{~A}$ & $22,2 \mathrm{~A}$ \\
\hline Diâmetro $(\mathrm{cm})$ & $2,7 \mathrm{~B}$ & $3,6 \mathrm{~A}$ & $2,5 \mathrm{~B}$ & $2,9 \mathrm{~A}$ & $2,9 \mathrm{~A}$ \\
\hline Matéria seca parte aérea $\left(\mathrm{g} \mathrm{kg}^{-1}\right)$ & $1,6 \mathrm{~B}$ & $5,7 \mathrm{~A}$ & $2,1 \mathrm{~B}$ & $3,1 \mathrm{~A}$ & $2,0 \mathrm{~A}$ \\
\hline Teor de $\mathrm{P}\left(\mathrm{g} \mathrm{kg}^{-1}\right)$ & $0,5 \mathrm{~B}$ & $1,5 \mathrm{~A}$ & $0,4 \mathrm{~B}$ & $0,8 \mathrm{~A}$ & $0,4 \mathrm{~A}$ \\
\hline Acúmulo de $\mathrm{P}\left(\mathrm{g}_{\text {planta }}{ }^{-1}\right)$ & $0,8 \mathrm{~B}$ & 7,9 A & $0,8 \mathrm{~B}$ & $3,2 \mathrm{~A}$ & $0,6 \mathrm{~A}$ \\
\hline Intensidade de colonização radicular (\%) & $0,4 \mathrm{~B}$ & $21,8 \mathrm{~A}$ & $0,2 \mathrm{~B}$ & $7,5 \mathrm{~A}$ & $0,0 \mathrm{~B}$ \\
\hline
\end{tabular}

Médias seguidas de letras iguais na linha não diferem entre si pelo teste de Skott e Knott a 5\% de probabilidade. 
Resultados semelhantes foram encontrados por Constantino et al. (2008), ao avaliarem o efeito de duas rizobactérias (Azotobacter chroococcum e Azospirillum brasilense) combinadas com várias espécies de FMAs sobre o crescimento e o rendimento de pimenta-chili (Capsicum chinense Jacquin). Os autores verificaram que, na fase de viveiro, a aplicação isolada de cada simbionte promoveu maior crescimento e teor de nutrientes na cultura do que quando combinado. Segundo os autores, esse resultado pode ser atribuído à não competição de cada simbionte por compostos de carbono, o que ocorreria quando em combinação, em que a planta hospedeira, além de necessitar de nutrientes, ainda compartilharia com os microrganismos simbiontes.

No que se refere à matéria fresca de raiz e número de esporos, também não houve diferença estatística entre o tratamento fatorial e o controle (Tabela 5). Quanto aos tratamentos, não houve efeito da interação entre as rizobactérias e FMAs, mas somente efeitos isolados tanto das rizobactérias quanto dos FMAs, com destaque para o FMA Acaulospora scrobiculata $\left(2,5 \mathrm{~g} \mathrm{planta}^{-1}\right)$ e rizobactérias Pseudomonas sp $\left(1,8 \mathrm{~g} \mathrm{planta}^{-1}\right)$ e Paenibacillus sp2 $\left(1,7 \mathrm{~g} \mathrm{planta}^{-1}\right)$ para matéria fresca de raiz e FMA Gigaspora rosea $\left(10,450 \mathrm{~mL}^{-1}\right)$ e Paenibacillus sp1 $\left(8,750 \mathrm{~mL}^{-1}\right)$ e Paenibacillus sp2 $\left(5,950 \mathrm{~mL}^{-1}\right)$ para o número de esporos.

\section{Tabela 5 - Efeito isolado dos FMAs e das rizobactérias na cultivar de oliveira Maria da Fé.}

Table 5 - Isolated effect of FMAs and rhizobacteria in the 'Maria da Fé' cultivar.

\begin{tabular}{|c|c|c|}
\hline & Matéria fresca de raiz $\left(\mathrm{g} \mathrm{planta}^{-1}\right)$ & Número de esporos (no $\left.50 \mathrm{~mL}^{-1}\right)$ \\
\hline Rhizophagus clarus & $0,7 \mathrm{~B}$ & $3,0 \mathrm{~B}$ \\
\hline Acaulospora scrobiculata & $2,5 \mathrm{~A}$ & $3,5 \mathrm{~B}$ \\
\hline Gigaspora rosea & $1,1 \mathrm{~B}$ & $10,4 \mathrm{~A}$ \\
\hline Pseudomonas sp & $1,8 \mathrm{~A}$ & $2,4 \mathrm{~B}$ \\
\hline Paenibacillus sp1 & $0,9 \mathrm{~B}$ & $8,7 \mathrm{~A}$ \\
\hline Paenibacillus sp2 & $1,7 \mathrm{~A}$ & $5,9 \mathrm{~A}$ \\
\hline Fatorial & $1,5 \mathrm{~A}$ & $5,7 \mathrm{~A}$ \\
\hline Controle & $1,3 \mathrm{~A}$ & $1,3 \mathrm{~A}$ \\
\hline
\end{tabular}

Médias seguidas de letras iguais na coluna não diferem entre si pelo teste de Skott e Knott a 5\% de probabilidade.

Nesse contexto, Sala, Freitas e Silveira (2007), ao avaliarem o efeito da coinoculação de dois isolados de bactérias diazotróficas (Achromobacter insolitus e Zoogloea ramigera) com duas espécies de FMAs (Glomus sp. e Acaulospora sp.) em trigo (Triticum durum L.)., tanto isoladamente quanto coinoculados, verificaram que, quando inoculadas as bactérias diazotróficas e os FMAs, isoladamente, houve maior crescimento, acúmulo e aproveitamento dos nutrientes por plantas de trigo. Esse resultado, segundo os autores, pode ter ocorrido em função de uma possível competição por compostos fotossintetizantes entre FMAs e bactérias, quando coinoculados, no período de colonização das raízes, na área cortical desta.

Assim como para a cultivar Arbequina, observou-se, de maneira geral, que houve efeito positivo da coinoculação para a cultivar de oliveira Maria da Fé, com incrementos no número de folhas e estado nutricional das mudas, como maior absorção de $\mathrm{N}$.

\section{Conclusões}

A coinoculação de fungos micorrízicos arbusculares e rizobactérias promove efeito diferenciado no crescimento e desenvolvimento de mudas das cultivares de oliveira Arbequina e Maria da Fé.

A combinação do FMA Acaulospora scrobiculata + Paenibacillus sp2 se destaca como positiva e com potencial de utilização para a produção de mudas das cultivares, principalmente para a Arbequina, por promover maior crescimento (altura e diâmetro) e produção de matéria fresca de raiz e seca da parte aérea. 
A cultivar Maria da Fé mostrou-se responsiva aos tratamentos de coinoculação, com incrementos no número de folhas e melhorias em seu estado nutricional, pelos maiores teores e acúmulos de nitrogênio na parte aérea e de porcentagem de colonização radicular e micélio extrarradicular total.

\section{Agradecimentos}

À Fundação de Amparo à Pesquisa de Minas Gerais (Fapemig), pelo apoio financeiro ao projeto APQ-02152-14 e à Coordenação de Aperfeiçoamento de Pessoal de Nível Superior (Capes), pela bolsa de mestrado concedida à primeira autora.

\section{Referências}

AGUIRRE-MEDINA, J. F.; MORENO, J. A. E. Crecimiento y rendimiento de Capsicum annuum L. inoculado com endomicorriza y rizobacterias. Revista Mexicana de Ciencias Agricolas, Texcoco, v. 7, n. 7, p. 1539-1550, 2016.

BALLUS, C. A. et al. A quantitative study on the phenolic compound, tocopherol and fatty acid contents of monovarietal virgin olive oils produced in the southeast region of Brazil. Food Research International, Ottawa, v. 62, n. 1, p. 74-83, 2014.

BATI, C. B.; SANTILLI, E.; LOMBARDO, L. Effect of arbuscular mycorrhizal fungi on growth and on micronutrient and macronutrient uptake and allocation in olive plantlets growing under high total Mn levels. Mycorrhiza, Berlin, v. 25, n. 2, p. 97-108, 2015.

BOMPADRE, M. J. et al. Evaluation of arbuscular mycorrhizal fungi capacity to alleviate abiotic stress of olive (Olea europaea L.) plants at different transplant conditions. The Scientific World Journal, London, v. 1, n. 1, p. 1-13, 2014.

BONA, E. et al. Arbuscular mycorrhizal fungi and plant growth-promoting pseudomonads improve yield, quality and nutritional value of tomato: a field study. Mycorrhiza, Berlin, v. 27, n. 1, p. 1-11, 2016.

CALVENTE, R. et al. Analisando a diversidade natural de fungos micorrízicos arbusculares em plantações de olivais (Olea europaea L.) e avaliação da eficácia de isolados de fungos nativos como inoculantes para cultivares comerciais de plantulas de oliveira. Applied Soil Ecology, Firenze, v. 26, n. 1, p. 11-19, 2004.

CARDOSO, E. J. B. N.; ANDREOTE, F. D. Microbiologia do solo. 2. ed. Piracicaba: ESALQ, 2016. 221 p.

CARVALHO, D. D. C. et al. Rizobactérias produtoras de promotores do crescimento de plantas. Pesquisa Agropecuária Tropical, Goiânia, v. 39, n. 4, p. 338-341, out./dez. 2009.

CONSTANTINO, M. et al. Effect of inoculation with rhizobacteria and arbuscular mycorrhizal fungi on growth and yield of Capsicum chinense Jacquin. Journal of Agriculture and Rural Development in the Tropics and Subtropics, Witzenhausen, v. 109, n. 2, p. 169-180, 2008.

COSTA, C. M. C. et al. Influência de fungos micorrízicos arbusculares sobre o crescimento de dois genótipos de aceroleira (Malpighia emarginata D.C.). Pesquisa Agropecuária Brasileira, Brasília, v. 36, n. 6, p. 893-901, jun. 2001.

COUTINHO, H. L. C. et al. Oliveira: aspectos técnicos e cultivo no sul do Brasil. 1. ed. Brasília: EMBRAPA, 2015.181 p.

DHAWI, F.; DATTA, R.; RAMAKRISHNA, W. Mycorrhiza and PGPB modulate maize biomass, nutrient uptake and metabolic pathways in maize grown in mining-impacted soil. Plant Physiology and Biochemistry, Bari, v. 97, n. 1, p. 390-399, 2015.

FERREIRA, E. B.; CAVALCANTI, P. P.; NOGUEIRA, D. A. Experimental designs: um pacote R para análise de experimentos. Revista da Estatística UFOP, Ouro Preto, v. 1, n. 1, p. 1-9, 2011. 
FERREIRA, G. M. R. et al. Fungos micorrízicos arbusculares no desenvolvimento de mudas de oliveira (Olea europaea L.) cultivadas no sul de Minas Gerais. Revista Brasileira de Ciência do Solo, Viçosa, MG, v. 39, n. 2, p. 361-366, 2015.

GERDEMANN, J. W.; NICOLSON, T. H. Spores of mycorrhizal Endogone species extracted from soil by wet sieving and decanting. Transactions of the British Mycological society, Cambridge, v. 46, n. 2, p. 235-244, 1963.

GIOVANNETTI, M.; MOSSE, B. An evaluation of techniques for measuring vesicular arbuscular mycorrhizal infection in roots. New Phytologist, Oxford, v. 84, n. 3, p. 489-500, 1980.

KIM, K. et al. Synergistic effects of inoculating arbuscular mycorrhizal fungi and Methylbacterium oryzae strains on growth and nutrient uptake of red pepper (Capsicum annuum L.). Plant and Soil, East Lansing, v. 327, n. 1/2, p. 429-440, 2010.

KUMAR, M. et al. Growth promoting characteristics of rhizobacteria and AM Fungi for biomass amelioration of Zea mays. Archives of Biological Sciences, Belgrado, v. 67, n. 3, p. 877-887, 2015.

LIMA, K. B. et al. Fungos micorrízicos arbusculares, bactérias diazotróficas e adubação fosfatada em mudas de mamoeiro. Revista Brasileira de Fruticultura, Jabotical, v. 33, n. 3, p. 932-940, 2011.

MALIK, N. S. A.; NUÑEZ, A.; MCKEEVER, L. C. Mycorrhizal inoculation increases growth and induces changes in specific polyphenol levels in olive saplings. Journal of Agricultural Science, Cambridge, v. 9, n. 2, p. 1, 2017.

MECHRI, B. et al. Changes in microbial communities and carbohydrate profiles induced by the mycorrhizal fungus (Glomus intraradices) in rhizosphere of olive trees (Olea europaea L.). Applied Soil Ecology, Firenze, v. 75, n. 1, p. 124-133, 2014.

MEDDAD-HAMZA, A. et al. Arbuscular mycorrhizal fungi improve the growth of olive trees and their resistance to transplantation stress. African Journal of Biotechnology, Kenya, v. 9, n. 8, p. 1159-1167, feb. 2010.

MEDDAD-HAMZA, A. et al. Spatiotemporal variation of arbuscular mycorrhizal fungal colonization in olive (Olea europaea L.) roots across a broad mesic-xeric climatic gradient in North Africa. Science of The Total Environment, Barcelona, v. 583, n. 1, p. 176-189, 2017.

MELLONI, R.; CARDOSO, E. J. B. N. Quantificação de micélio extrarradicular de fungos micorrízicos arbusculares em plantas cítricas. II. Comparação entre diferentes espécies cítricas e endófitos. Revista Brasileira de Ciência do Solo, Viçosa, MG, v. 23, n. 1, p. 59-67, 1999.

MONTERO-CALASANZ, M. C. et al. Alternative rooting induction of semi-hardwood olive cuttings by several auxin-producing bacteria for organic agriculture systems. Spanish Journal of Agricultural Research, Madrid, v. 11, n. 1, p. 146-154, 2013.

MONTES-BORREGO, M.; METSIS, M.; LANDA, B. B. Arbuscular mycorhizal fungi associated with the olive crop across the andalusian landscape: factors community differentiation. PlosOne, San Francisco, v. 9, n. 5, p. 1-12, may 2014.

OLIVEIRA, J. R. G. et al. Fungos micorrízicos arbusculares e rizobactérias promotoras de crescimento na aclimatização de Zingiber spectabile. Bragantia, Campinas, v. 69, n. 3, p. 687-694, 2010.

OLIVEIRA, M. C. et al. Características fenológicas e físicas e perfil de ácidos graxos em oliveiras no sul de Minas Gerais. Pesquisa Agropecuária Brasileira, Brasília, v. 47, n. 1, p. 30-35, jan. 2012.

PAIS, A. K. L. et al. Seleção de rizobactérias como promotoras de crescimento em melancia. Scientia Plena, São Cristóvão, v. 12, n. 4, p. 1-8, 2016.

PEYVANDI, M. et al. Pseudomonas fluorescent and its ability to promote root formation of olive microshoots. International Journal of Plant Production, Gorgan, v. 4, n. 1, p. 63-66, jan. 2010. 
PHILLIPS, J. M.; HAYMAN, D. S. Improved procedures for clearing roots and staining parasitic and vesicular-arbuscular mycorrhizal fungi for rapid assessment of infection. Transactions of the British Mycological Society, Cambridge, v. 55, n. 1, p. 158IN16-161IN18, 1970.

RAMASAMY, K. et al. Synergistic effects of arbuscular mycorrhizal fungi and plant growth promoting rhizobacteria for sustainable agricultural production. Korean Journal of Soil Science and Fertilizer, Jeollabuk-do, v. 44, n. 4, p. 637-649, 2011.

RODRÍGUEZ-ROMERO, A. S.; GUERRA, M. S. P.; JAIZME-VEGA, M. D. C. Effect of arbuscular mycorrhizal fungi and rhizobacteria on banana growth and nutrition. Agronomy for Sustainable Development, Paris, v. 25, n. 3, p. 395-399, 2005.

SALA, V. M. R.; FREITAS, S. S.; SILVEIRA, A. P. D. Interação de fungos micorrízicosarbusculares e bactérias diazotróficas em trigo. Pesquisa Agropecuária Brasileira, Brasília, v. 42, n. 11, p. 1593-1600, 2007.

SEIFI, E. et al. Olive mycorrhization: Influences of genotype, mycorrhiza, and growing periods. Scientia Horticulturae, Columbia, v. 180, n. 1, p. 214-219, 2014.

SILVA, T. F. et al. Bactérias diazotróficas não simbióticas e enraizamento de estacas semilenhosas de oliveira (Olea europaea L.). Ciência Florestal, Santa Maria, v. 27, n. 1, p. 61-71, jan./mar. 2017.

SMITH, S. E.; READ, D. J. Mycorrhizal symbiosis. London: Academic Press, 2008.

TEKAYA, M. et al. Arbuscular mycorrhizal fungus Rhizophagus irregularis influences key physiological parameters of olive trees (Olea europaea L.) and mineral nutrient profile, Photosynthetica, Praha, v. 55, n .2, p. 308-316, 2017.

VALADARES, R. B. S.; MESCOLOTTI, D. L. C.; CARDOSO, E. J. B. N. Micorrizas. In: CARDOSO, E. J. B. N.; ANDREOTE, F. D. Microbiologia do Solo. Piracicaba: ESALQ, 2016. p. 179-196.

VEJAN, P et al. Role of plant growth promoting rhizobacteria in agricultural sustainability - a review. Molecules, Basel, v. 21, n. 5, p. 573, 2016.

VIEIRA NETO, J. et al. Aspectos técnicos da cultura da oliveira. 1. ed. Belo Horizonte: Empresa de Pesquisa Agropecuária de Minas Gerais, 2008. 56 p.

VIEIRA, V. C. S.; MELLONI, R.; VIEIRANETO, J. Avaliação da interação micorrízica em cultivares de oliveira (Olea europaea L.). Revista Brasileira de Ciência do Solo, Viçosa, MG, v. 35, n. 6, p. 1885-1892, 2011.

WEBER, O. B.; AMORIM, A. M. C. Adubação fosfática e inoculação de fungos micorrízicos vesículo-arbusculares em mamoeiro "Solo". Revista Brasileira de Ciência do Solo, Campinas, v. 18, n. 2, p. 187-191, 1994. 